Jun $Y e^{*}$

\title{
Single-Valued Neutrosophic Minimum Spanning Tree and Its Clustering Method
}

\begin{abstract}
Clustering plays an important role in data mining, pattern recognition, and machine learning. Then, single-valued neutrosophic sets (SVNSs) are a useful means to describe and handle indeterminate and inconsistent information, which fuzzy sets and intuitionistic fuzzy sets cannot describe and deal with. To cluster the data represented by single-value neutrosophic information, the article proposes a single-valued neutrosophic minimum spanning tree (SVNMST) clustering algorithm. Firstly, we defined a generalized distance measure between SVNSs. Then, we present an SVNMST clustering algorithm for clustering single-value neutrosophic data based on the generalized distance measure of SVNSs. Finally, two illustrative examples are given to demonstrate the application and effectiveness of the developed approach.
\end{abstract}

Keywords: Neutrosophic set, single-valued neutrosophic set, minimum spanning tree, clustering algorithm, generalized distance measure.

*Corresponding author: Jun Ye, Department of Electrical and Information Engineering, Shaoxing University, 508 Huancheng West Road, Shaoxing, Zhejiang Province 312000, P.R. China, Phone: +86-575-88327323, e-mail: yehjun@aliyun.com

\section{Introduction}

Clustering plays an important role in data mining, pattern recognition, and machine learning. Clustering data sets into disjoint groups is a problem arising in many domains. Generally, the goal of clustering is to find groups that are both homogeneous and well separated, that is, entities within the same group should be similar and entities in different groups dissimilar. Then, data sets can be represented by weighted graphs, where nodes correspond to the entities to be clustered and edges correspond to a dissimilarity or similarity measure between those entities. Graph theory [3] provided us with a very convenient tool to describe clustering problems. However, a minimum spanning tree (MST) is a very useful graph structure and can capture perceptual grouping [4]. Zahn [13] defined several criteria of edge inconsistency for detecting clusters of different shapes and proposed the clustering algorithm using MST. Xu et al. [10] introduced three MST algorithms and applied them to clustering gene expression data. Owing to the fuzziness and uncertainty of many practical problems in the real world, Ruspini [7] first presented the concept of fuzzy division and a fuzzy clustering approach. Dong et al. [2] introduced a hierarchical clustering algorithm based on fuzzy graph connectedness. Then, Chen et al. [1] put forward a fuzzy graph maximal tree clustering method of the fuzzy graph constructing the fuzzy similarity relation matrix and used the threshold of fuzzy similarity relation matrix to cut maximum spanning tree, and then obtained the classification on level. Zhao et al. [15] proposed two intuitionistic fuzzy minimum spanning tree (IFMST) clustering algorithms to deal with intuitionistic fuzzy information and extended them to clustering interval-valued intuitionistic fuzzy information. Furthermore, Zhang and Xu [14] introduced a MST algorithm-based clustering method under hesitant fuzzy environment.

To represent uncertainty, imprecise, incomplete, and inconsistent information that exist in real world, Smarandache [8] gave the concept of a neutrosophic set from philosophical point of view. The neutrosophic set [8] is a powerful general formal framework that generalizes the concept of the classic set, fuzzy set, interval valued fuzzy set, intuitionistic fuzzy set, interval valued intuitionistic fuzzy set, paraconsistent set, dialetheist set, paradoxist set, and tautological set. In the neutrosophic set, truth membership, indeterminacy membership, and falsity membership are represented independently. However, the neutrosophic set 
generalizes the above-mentioned sets from the philosophical point of view, and its functions $T_{A}(x), I_{A}(x)$, and $F_{A}(x)$ are real standard or nonstandard subsets of ]-0, $1^{+}\left[\text {, i.e., } T_{A}(x): X \rightarrow\right]^{-} 0,1^{+}\left[, I_{A}(x): X \rightarrow\right]^{-} 0,1^{+}[$, and $\left.F_{A}(x): X \rightarrow\right]^{-} 0,1^{+}[$. So it will be difficult to apply in real scientific and engineering areas. Thus, Wang et al. [9] introduced a single-valued neutrosophic set (SVNS), which is an instance of a neutrosophic set. It can describe and handle indeterminate information and inconsistent information, which fuzzy sets and intuitionistic fuzzy sets cannot describe and deal with. Recently, Ye [11, 12] presented the correlation coefficient of SVNSs and the cross-entropy measure of SVNSs and applied them to single-valued neutrosophic decision-making problems. Yet, until now, there has been no study on clustering the data represented by single-valued neutrosophic information. However, the existing MST clustering algorithms cannot cluster the single-valued neutrosophic data. Therefore, this article proposes a single-valued neutrosophic minimum spanning tree (SVNMST) clustering algorithm to deal with the data represented by SVNSs. To do so, the rest of the article is organized as follows. Section 2 introduces some basic concepts of SVNSs, the graph, and its MST. Section 3 defines a generalized distance measure between SVNSs. In Section 4, a single-valued neutrosophic clustering algorithm is proposed based on the MST. In Section 5, two illustrative examples are given to demonstrate the applications and the effectiveness of the proposed approach. Conclusions and further research are contained in Section 6.

\section{Preliminaries}

In this section, some basic concepts of SVNSs, the graph, and its MST are introduced to be utilized in the next sections.

\subsection{Some Concepts of SVNSs}

Smarandache [8] introduced the concept of a neutrosophic set from philosophical point of view.

Definition 1 [8]. Let $X$ be a space of points (objects), with a generic element in $X$ denoted by $x$. A neutrosophic set $A$ in $X$ is characterized by a truth membership function $T_{A}(x)$, an indeterminacy membership function $I_{A}(x)$, and a falsity membership function $F_{A}(x)$. The functions $T_{A}(x), I_{A}(x)$, and $F_{A}(x)$ are real standard or nonstandard subsets of $]^{-} 0,1^{+}\left[\text {, that is, } T_{A}(x): X \rightarrow\right]^{-} 0,1^{+}\left[, I_{A}(x): X \rightarrow\right]^{-} 0,1^{+}\left[\text {, and } F_{A}(x): X \rightarrow\right]^{-} 0$, $1^{+}[$, with the condition ${ }^{-} 0 \leq \sup T_{A}(x)+\sup I_{A}(x)+\sup F_{A}(x) \leq 3^{+}$.

Obviously, it is difficult to apply in real scientific and engineering fields. Thus, Wang et al. [9] introduced the concept of an SVNS, which is an instance of a neutrosophic set.

Definition 2 [9]. Let $X$ be a space of points (objects) with generic elements in $X$ denoted by $x$. An SVNS $A$ in $X$ is characterized by truth membership function $T_{A}(x)$, indeterminacy membership function $I_{A}(x)$, and falsity membership function $F_{A}(x)$. For each point $x$ in $X$, there are $T_{A}(x), I_{A}(x), F_{A}(x) \in[0,1]$, and $0 \leq T_{A}(x)+I_{A}(x)+$ $F_{A}(x) \leq 3$. Therefore, an SVNS $A$ can be represented by

$$
A=\left\{\left\langle x, T_{A}(x), I_{A}(x), F_{A}(x)\right\rangle \mid x \in X\right\} .
$$

The following expressions are defined in [9] for SVNSs $A, B$ :

1. $A \subseteq B$ if and only if $T_{A}(x) \leq T_{B}(x), I_{A}(x) \geq I_{B}(x), F_{A}(x) \geq F_{B}(x)$ for any $x$ in $X$.

2. $A=B$ if and only if $A \subseteq B$ and $B \subseteq A$.

3. $A^{c}=\left\{\left\langle x, F_{A}(x), 1-I_{A}(x), T_{A}(x)\right\rangle \mid x \in X\right\}$. 


\subsection{Graph and MSTs}

A graph $G$ is a pair of sets $G=(V, E)$, where $V$ is the set of vertices (or nodes) and $E$ is the set of edges. There are two kinds of graphs: an undirected graph and a directed graph. Each edge in the undirected graph is an unordered pair $\left\{v_{i}, v_{j}\right\}$, whereas each edge in the directed graph is an ordered pair $\left\{v_{i}, v_{j}\right\}$, where the vertices $v_{i}$ and $v_{j}$ are called the end points of an edge. A sequence of edges and vertices that can be traveled between two different vertices is called a path. Suppose five nodes are given, then a graph with five nodes and seven edges is shown in Figure 1.

In Figure 1, there are different paths from the node $B$ to the node $E$, such as the path (BAE) and the other path (BCDE). For a path, the start node and the destination node are the same, which is called a circuit, like (ABCA) or (ADEA).

A connected graph has paths between any pair of nodes. A connected acyclic graph that contains all nodes of $G$ is called a spanning tree of the graph, which is any set of straight line segments connecting pairs of nodes such that

1. no closed loops occur,

2. each node is visited by at least one line, and

3. a tree is connected.

For example, a spanning tree with integer segment lengths is shown in Figure 2, which is obtained from Figure 1. When the nodes $B$ and $C$ or $D$ and $E$ were connected, a closed loop would be formed and the resulting figure would not be a tree. Then, we define the length of a tree to be the sum of the lengths of its constituent edges, the length of the tree in Figure 2 is $4+6+5+8=23$ units.

An MST is the spanning tree of the minimum length that is often required. For instance, Figure 3 shows the MST obtained from Figure 1, and then its minimum length is $4+3+2+5=14$ units.

Various algorithms to find the MST have been proposed in $[5,6,10]$. However, there are the two most popular algorithms to find the MST of a graph $G$ by operating iteratively $[5,6]$, which are needed later. At any stage, the segment belongs to one of two sets, i.e., set $A$ containing those segments assigned to the MST and set $B$, those not assigned.

The algorithm given by Kruskal [5] is to assign interactively to set $A$ the shortest segment in the set $B$, which does not form a closed loop with any of the segments already in $A$. Initially, $A$ is empty, and the iteration stops when $A$ contains $(n-1)$ segments.

The algorithm given by Prim [6] starts with any one of the given nodes and initially assign to $A$ the shortest segment starting from this node. The procedure continues to add the shortest segment from $B$, which connects to at least one segment from $A$ without forming a closed loop among the segments already in $A$. The iteration stops when $A$ contains $(n-1)$ segments.

Usually, clustering data sets can be represented as weighted graphs, where nodes correspond to the entities to be clustered and edges correspond to distance measure (or called dissimilarity measure) between

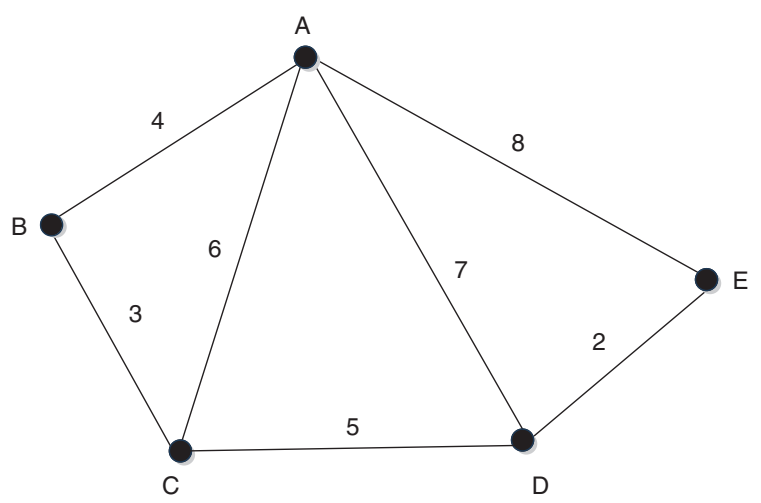

Figure 1. Graph. 


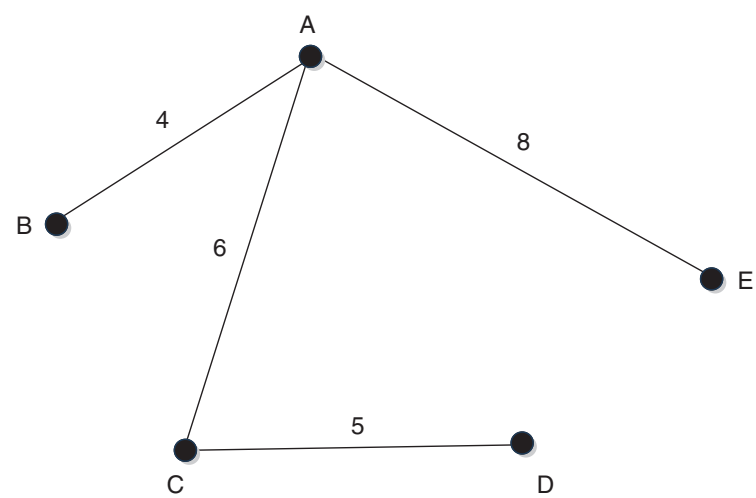

Figure 2. Spanning Tree.

those entities. If a fuzzy relation $R$ over $V \times V$ is defined, then the membership function $\mu_{R}\left(v_{1}, v_{2}\right)$, where $\left(v_{1}\right.$, $\left.v_{2}\right) \in V \times V$ takes various values from 0 to 1 , and then such a graph is called a fuzzy graph. When $R$ is an intuitionistic fuzzy relation over $V \times V$, then such a graph is called an intuitionistic fuzzy graph [15].

\section{Distance Measures of SVNSs}

For two SVNSs $A$ and $B$ in a universe of discourse, $X=\left\{x_{1}, x_{2}, \ldots, x_{n}\right\}$, which are denoted by $A=\left\{\left\langle x_{i}, T_{A}\left(x_{i}\right), I_{A}\left(x_{i}\right)\right.\right.$, $\left.\left.F_{A}\left(x_{i}\right)\right\rangle \mid x_{i} \in X\right\}$, and $B=\left\{\left\langle x_{i}, T_{A}\left(x_{i}\right), I_{A}\left(x_{i}\right), F_{A}\left(x_{i}\right)\right\rangle \mid x_{i} \in X\right\}$, where $T_{A}\left(x_{i}\right), I_{A}\left(x_{i}\right), F_{A}\left(x_{i}\right), T_{B}\left(x_{i}\right), I_{B}\left(x_{i}\right), F_{B}\left(x_{i}\right) \in[0,1]$ for every $x_{i} \in X$. Let us to consider the weight $w_{i}(i=1,2, \ldots, n)$ of an element $x_{i}(i=1,2, \ldots, n)$, with $w_{i} \geq 0(i=1,2, \ldots, n)$ and $\sum_{i=1}^{n} w_{i}=1$. Then, we define the generalized single-valued neutrosophic-weighted distance:

$$
d_{\lambda}(A, B)=\left\{\frac{1}{3} \sum_{i=1}^{n} w_{i}\left[\left|T_{A}\left(x_{i}\right)-T_{B}\left(x_{i}\right)\right|^{\lambda}+\left|I_{A}\left(x_{i}\right)-I_{B}\left(x_{i}\right)\right|^{\lambda}+\left|F_{A}\left(x_{i}\right)-F_{B}\left(x_{i}\right)\right|^{\lambda}\right]\right\}^{1 / \lambda},
$$

where $\lambda>0$.

Especially if $\lambda=1,2$, Equation (1) reduces to the single-valued neutrosophic-weighted Hamming distance and the single-valued neutrosophic-weighted Euclidean distance, respectively, as follows:

$$
d_{1}(A, B)=\frac{1}{3} \sum_{i=1}^{n} w_{i}\left[\left|T_{A}\left(x_{i}\right)-T_{B}\left(x_{i}\right)\right|+\left|I_{A}\left(x_{i}\right)-I_{B}\left(x_{i}\right)\right|+\left|F_{A}\left(x_{i}\right)-F_{B}\left(x_{i}\right)\right|\right],
$$

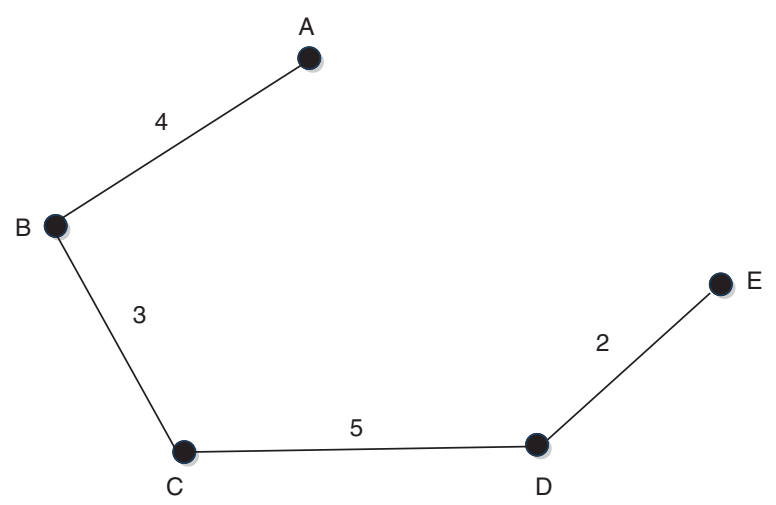

Figure 3. Minimum Spanning Tree. 


$$
d_{2}(A, B)=\left\{\frac{1}{3} \sum_{i=1}^{n} w_{i}\left[\left|T_{A}\left(x_{i}\right)-T_{B}\left(x_{i}\right)\right|^{2}+\left|I_{A}\left(x_{i}\right)-I_{B}\left(x_{i}\right)\right|^{2}+\left|F_{A}\left(x_{i}\right)-F_{B}\left(x_{i}\right)\right|^{2}\right]\right\}^{1 / 2} .
$$

Then, for the distance measure we have the following proposition.

Proposition 1. The above-defined distance $d_{\lambda}(A, B)$ for $\lambda>0$ satisfies the following properties:

(P1) $d_{\lambda}(A, B) \geq 0$;

(P2) $d_{\lambda}(A, B)=0$ if and only if $A=B$;

(P3) $d_{\lambda}(A, B)=d_{\lambda}(B, A)$;

(P4) If $A \subseteq B \subseteq C, C$ is an SVNS in $X$, then $d_{\lambda}(A, C) \geq d_{\lambda}(A, B)$ and $d_{\lambda}(A, C) \geq d_{\lambda}(B, C)$.

Proof. It is easy to see that $d_{\lambda}(A, B)$ satisfies the properties (P1)-(P3). Therefore, we only prove (P4). Let $A \subseteq$ $B \subseteq C$, then, $T_{A}\left(x_{i}\right) \leq T_{B}\left(x_{i}\right) \leq T_{C}\left(x_{i}\right), I_{A}\left(x_{i}\right) \geq I_{B}\left(x_{i}\right) \geq I_{C}\left(x_{i}\right)$, and $F_{A}\left(x_{i}\right) \geq F_{B}\left(x_{i}\right) \geq F_{C}\left(x_{i}\right)$ for every $x_{i} \in X$. Then, we obtain the following relations:

$$
\begin{aligned}
& \left|T_{A}\left(x_{i}\right)-T_{B}\left(x_{i}\right)\right|^{\lambda} \leq\left|T_{A}\left(x_{i}\right)-T_{C}\left(x_{i}\right)\right|^{\lambda},\left|T_{B}\left(x_{i}\right)-T_{C}\left(x_{i}\right)\right|^{\lambda} \leq\left|T_{A}\left(x_{i}\right)-T_{C}\left(x_{i}\right)\right|^{\lambda}, \\
& \left|I_{A}\left(x_{i}\right)-I_{B}\left(x_{i}\right)\right|^{\lambda} \leq\left|I_{A}\left(x_{i}\right)-I_{C}\left(x_{i}\right)\right|^{\lambda},\left|I_{B}\left(x_{i}\right)-I_{C}\left(x_{i}\right)\right|^{\lambda} \leq\left|I_{A}\left(x_{i}\right)-I_{C}\left(x_{i}\right)\right|^{\lambda}, \\
& \left|F_{A}\left(x_{i}\right)-F_{B}\left(x_{i}\right)\right|^{\lambda} \leq\left|F_{A}\left(x_{i}\right)-F_{C}\left(x_{i}\right)\right|^{\lambda},\left|F_{B}\left(x_{i}\right)-F_{C}\left(x_{i}\right)\right|^{\lambda} \leq\left|F_{A}\left(x_{i}\right)-F_{C}\left(x_{i}\right)\right|^{\lambda} .
\end{aligned}
$$

Hence,

$$
\begin{aligned}
& \left|T_{A}\left(x_{i}\right)-T_{B}\left(x_{i}\right)\right|^{\lambda}+\left|I_{A}\left(x_{i}\right)-I_{B}\left(x_{i}\right)\right|^{\lambda}+\left|F_{A}\left(x_{i}\right)-F_{B}\left(x_{i}\right)\right|^{\lambda} \leq\left|T_{A}\left(x_{i}\right)-T_{C}\left(x_{i}\right)\right|^{\lambda}+\left|I_{A}\left(x_{i}\right)-I_{C}\left(x_{i}\right)\right|^{\lambda}+\left|F_{A}\left(x_{i}\right)-F_{C}\left(x_{i}\right)\right|^{\lambda}, \\
& \left|T_{B}\left(x_{i}\right)-T_{C}\left(x_{i}\right)\right|^{\lambda}+\left|I_{B}\left(x_{i}\right)-I_{C}\left(x_{i}\right)\right|^{\lambda}+\left|F_{B}\left(x_{i}\right)-F_{C}\left(x_{i}\right)\right|^{\lambda} \leq\left|T_{A}\left(x_{i}\right)-T_{C}\left(x_{i}\right)\right|^{\lambda}+\left|I_{A}\left(x_{i}\right)-I_{C}\left(x_{i}\right)\right|^{\lambda}+\left|F_{A}\left(x_{i}\right)-F_{C}\left(x_{i}\right)\right|^{\lambda} .
\end{aligned}
$$

Combining the above inequalities with the above-defined distance formula (1), we can obtain that

$$
d_{\lambda}(A, B) \leq d_{\lambda}(A, C) \text { and } d_{\lambda}(B, C) \leq d_{\lambda}(A, C) \text { for } \lambda>0 .
$$

Thus, the property (P4) is obtained.

In the following, we give the definition of single-valued neutrosophic distance matrix.

Definition 3. Let $A_{j}(j=1,2, \ldots, m)$ be a collection of $m$ SVNSs, then $D=\left(d_{i j}\right)_{m \times m}$ is called a single-valued neutrosophic distance matrix, where $d_{i j}=d_{\lambda}\left(A_{i}, A_{j}\right)$ is the distance between $A_{i}$ and $A_{j}$, and its properties are as follows:

1. $0 \leq d_{i j} \leq 1$ for all $i, j=1,2, \ldots, m$;

2. $\quad d_{i j}=0$ if and only if $A_{i}=A_{j}$;

3. $d_{i j}=d_{j i}$ for all $i, j=1,2, \ldots, m$.

\section{SVNMST Clustering Algorithm}

In this section, an SVNMST clustering algorithm is proposed as a generalization of an IFMST clustering algorithm [15].

Let $X=\left\{x_{1}, x_{2}, \ldots, x_{n}\right\}$ be an attribution space and the weight vector of an element $x_{i}(i=1,2, \ldots, n)$ be $w=$ $\left\{w_{1}, w_{2}, \ldots, w_{n}\right\}$, with $w_{i} \geq 0(i=1,2, \ldots, n)$ and $\sum_{i=1}^{n} w_{i}=1$. Assume that $A_{j}(j=1,2, \ldots, m)$ is a collection of $m$ SVNSs, which express $m$ samples to be clustered. Then, there is the following form: 


$$
A_{j}=\left\{\left\langle x_{i}, T_{A_{j}}\left(x_{i}\right), I_{A_{j}}\left(x_{i}\right), F_{A_{j}}\left(x_{i}\right)\right\rangle \mid x_{i} \in X\right\}
$$

Thus, we propose an SVNMST clustering algorithm, which is described by the following steps:

Step 1. Calculate the distance of $d_{i j}=d_{\lambda}\left(A_{i}, A_{j}\right)(i, j=1,2, \ldots, m)$ by Equation (1) to establish the single-valued neutrosophic distance matrix $D=\left(d_{i j}\right)_{m \times m}$.

Step 2. Draw the single-valued neutrosophic graph $G(V, E)$ where every edge between $A_{i}$ and $A_{j}$ is assigned the weight (single-valued neutrosophic distance) $d_{i j}$ coming from an element of the single-valued neutrosophic distance matrix $D=\left(d_{i j}\right)_{m \times m}$, which represents the dissimilarity degree between samples $A_{i}$ and $A_{j}$.

Step 3. Build the MST of the single-valued neutrosophic graph $G(V, E)$ by Kruskal's method [5] or Prim's method [6]:

1. Sort the edges of $G$ in increasing order by weights.

2. Keep a subgraph $S$ of $G$, which is initially empty, and at each step, choose the edge $e$ with the smallest weight to be added to the subgraph $S$, where the end point of $e$ are disconnected.

3. Repeat process (2) until the subgraph $S$ spans all vertices. Hence, the MST of the single-valued neutrosophic graph $G(V, E)$ is obtained.

Step 4. Perform clustering by use of the SVNMST. We can get a certain number of subtrees (clustering) by disconnecting all the edges of the MST with weights greater than a threshold $r$. The clustering results induced by the subtrees do not depend on some particular MST [14, 15].

\section{Illustrative Examples}

In this section, two illustrative examples are presented to demonstrate the real applications and the effectiveness of the proposed approach.

Example 1. A car market is going to classify eight different cars of $A_{j}(j=1,2, \ldots, 8)$. Every car has six evaluation factors (attributes): (1) $x_{1}$ : fuel consumption; (2) $x_{2}$ : coefficient of friction; (3) $x_{3}$ : price; (4) $x_{4}$ : comfortable degree; (5) $x_{5}$ : design; (6) $x_{6}$ : security coefficient. The characteristics of each car under the six attributes are represented by the form of SVNSs, and then the single-valued neutrosophic data are as follows:

$A_{1}=\left\{<x_{1}, 0.3,0.2,0.5>,<x_{2}, 0.6,0.3,0.1>,<x_{3}, 0.4,0.3,0.3>,<x_{4}, 0.8,0.1,0.1>,<x_{5}, 0.1,0.3,0.6>,<x_{6}, 0.5\right.$, $0.2,0.4>\}$

$A_{2}=\left\{<x_{1}, 0.6,0.3,0.3>,<x_{2}, 0.5,0.4,0.2>,<x_{3}, 0.6,0.2,0.1>,<x_{4}, 0.7,0.2,0.1>,<x_{5}, 0.3,0.1,0.6>,<x_{6}, 0.4\right.$, $0.3,0.3>\}$;

$A_{3}=\left\{<x_{1}, 0.4,0.2,0.4>,<x_{2}, 0.8,0.2,0.1>,<x_{3}, 0.5,0.3,0.1>,<x_{4}, 0.6,0.1,0.2>,<x_{5}, 0.4,0.1,0.5>,<x_{6}, 0.3\right.$, $0.2,0.2>\}$

$$
A_{4}=\left\{<x_{1}, 0.2,0.4,0.4>,<x_{2}, 0.4,0.5,0.1>,<x_{3}, 0.9,0.2,0.0>,<x_{4}, 0.8,0.2,0.1>,<x_{5}, 0.2,0.3,0.5>,<x_{6}, 0.7,\right.
$$
$0.3,0.1>\}$;

$$
A_{5}=\left\{<x_{1}, 0.2,0.3,0.3>,<x_{2}, 0.3,0.2,0.6>,<x_{3}, 0.5,0.1,0.4>,<x_{4}, 0.7,0.1,0.1>,<x_{5}, 0.4,0.2,0.4>,<x_{6}, 0.3,\right.
$$

$0.2,0.6>\}$;

$A_{6}=\left\{<x_{1}, 0.3,0.2,0.4>,\left\langle x_{2}, 0.2,0.1,0.7>,\left\langle x_{3}, 0.4,0.2,0.5\right\rangle,\left\langle x_{4}, 0.8,0.0,0.1>,<x_{5}, 0.4,0.3,0.5>,<x_{6}, 0.2\right.\right.\right.$, $0.1,0.7>\}$;

$A_{7}=\left\{<x_{1}, 0.4,0.4,0.3>,<x_{2}, 0.5,0.3,0.1>,<x_{3}, 0.6,0.1,0.2>,<x_{4}, 0.2,0.3,0.7>,<x_{5}, 0.3,0.1,0.5>,<x_{6}, 0.7\right.$, $0.2,0.1>\}$;

$A_{8}=\left\{<x_{1}, 0.4,0.1,0.2>,<x_{2}, 0.6,0.1,0.1>,<x_{3}, 0.8,0.2,0.1>,<x_{4}, 0.7,0.2,0.1>,<x_{5}, 0.1,0.1,0.8>,<x_{6}, 0.2\right.$, $0.1,0.8>\}$.

If the weight vector of the attribute $x_{i}(i=1,2, \ldots, 6)$ is $w=(0.16,0.12,0.25,0.2,0.15,0.12)^{\mathrm{T}}$, then we utilize the SVNMST clustering algorithm to group the eight different cars of $A_{j}(j=1,2, \ldots, 8)$. 
Step 1. Calculate the distance $d_{i j}=d_{\lambda}\left(A_{i}, A_{j}\right)$ by Equation (1) (take $\left.\lambda=2\right)$. Then we can establish the singlevalued neutrosophic distance matrix $D=\left(d_{i j}\right)_{m \times m}$ as follows:

$$
D=\left[\begin{array}{cccccccc}
0.0000 & 0.2737 & 0.2551 & 0.3583 & 0.4022 & 0.4559 & 0.5052 & 0.4100 \\
0.2737 & 0.0000 & 0.2269 & 0.2605 & 0.4064 & 0.5334 & 0.4271 & 0.4117 \\
0.2551 & 0.2269 & 0.0000 & 0.3311 & 0.4577 & 0.5527 & 0.3983 & 0.4450 \\
0.3583 & 0.2605 & 0.3311 & 0.0000 & 0.5442 & 0.6720 & 0.4559 & 0.5794 \\
0.4022 & 0.4064 & 0.4577 & 0.5442 & 0.0000 & 0.1987 & 0.5957 & 0.4811 \\
0.4559 & 0.5334 & 0.5527 & 0.6720 & 0.1987 & 0.0000 & 0.7155 & 0.5248 \\
0.5052 & 0.4271 & 0.3983 & 0.4559 & 0.5957 & 0.7155 & 0.0000 & 0.6370 \\
0.4100 & 0.4117 & 0.4450 & 0.5794 & 0.4811 & 0.5248 & 0.6370 & 0.0000
\end{array}\right] .
$$

Step 2. Draw the single-valued neutrosophic graph $G(V, E)$ where every edge between $A_{i}$ and $A_{j}(i, j=1,2, \ldots, 8)$ is assigned the weight (single-valued neutrosophic distance) $d_{i j}$ coming from an element of the singlevalued neutrosophic distance matrix $D=\left(d_{i j}\right)_{m \times m}$, which represents the dissimilarity degree between the samples $A_{i}$ and $A_{i}$. Then, the single-valued neutrosophic graph $G(V, E)$ is shown in Figure 4.

Step 3. Establish the MST of the single-valued neutrosophic graph $G(V, E)$ by Kruskal's method [5] or Prim's method [6]:

1. Sort the edges of $G$ in increasing order by weights:

$d_{56}<d_{23}<d_{13}<d_{24}<d_{12}<d_{34}<d_{14}<d_{37}<d_{15}<d_{25}<d_{18}<d_{28}<d_{27}<d_{38}<d_{16}=d_{47}<d_{35}<d_{58}<d_{17}<d_{68}<$ $d_{26}<d_{45}<d_{36}<d_{48}<d_{57}<d_{78}<d_{46}<d_{67}$

2. Keep an empty subgraph $S$ of $G$ and add the edge $e$ with the smallest weight to $S$, where the end points of $e$ are disconnected; thus, we choose the edge $e_{56}$ between $A_{5}$ and $A_{6}$.

3. Repeat process (2) until the subgraph $S$ spans eight nodes. Thus, the MST of the single-valued neutrosophic graph $G(V, E)$ is obtained, as shown in Figure 5.

Step 4. Select a threshold $r$ and disconnect all the edges of the MST with weights greater than $r$ to obtain a certain number of subtrees (clusters), as listed in Table 1.

To compare the SVNMST clustering algorithm with the intuitionistic fuzzy MST clustering algorithm and the fuzzy MST clustering algorithm, we introduce the following example discussed in $[14,15]$ for comparative convenience.

Example 2. To complete an operational mission, the six sets of operational plans are made (adapted from $[14,15])$. To group these operational plans with respect to their comprehensive function, a military committee

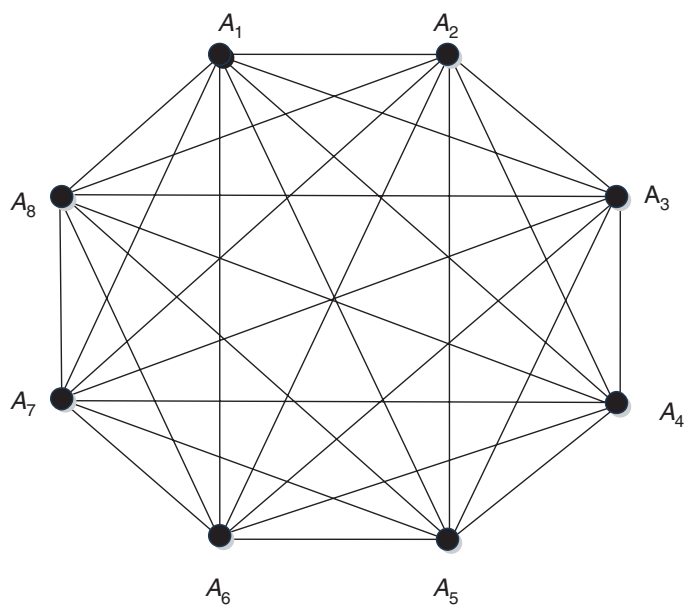

Figure 4. Single-Valued Neutrosophic Graph $G(V, E)$ with the Eight Nodes. 


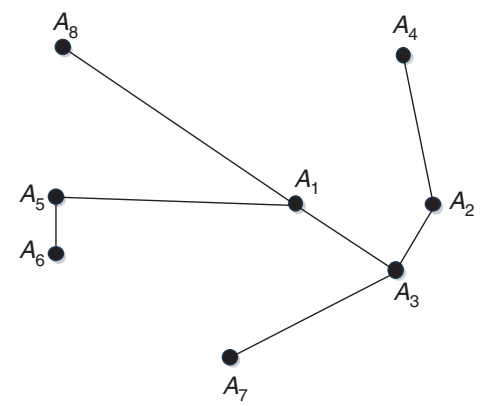

Figure 5. The MST of the Single-Valued Neutrosophic Graph $G(V, E)$ with the Eight Nodes.

has been set up to provide assessment information on them. The attributes that are considered here in assessment of the six operational plans $A_{j}(j=1,2, \ldots, 6)$ are (1) $x_{1}$ is the effectiveness of operational organization and (2) $x_{2}$ is the effectiveness of operational command. The weight vector of the attributes $x_{i}(i=1,2)$ is $w=$ $(0.45,0.55)^{\mathrm{T}}$. The military committee evaluates the performance of the six operational plans $A_{j}(j=1,2, \ldots, 6)$ with respect to the attributes $x_{i}(i=1,2)$ and gives the SVNSs as follows:

$$
\begin{gathered}
A_{1}=\left\{<x_{1}, 0.7,0.2,0.15>,<x_{2}, 0.6,0.3,0.2>\right\} ; \\
A_{2}=\left\{<x_{1}, 0.4,0.3,0.35>,<x_{2}, 0.8,0.1,0.1>\right\} ; \\
A_{3}=\left\{<x_{1}, 0.55,0.2,0.25>,<x_{2}, 0.7,0.1,0.15>\right\} ; \\
A_{4}=\left\{<x_{1}, 0.44,0.2,0.35>,<x_{2}, 0.6,0.2,0.2>\right\} ; \\
A_{5}=\left\{<x_{1}, 0.5,0.15,0.35>,<x_{2}, 0.75,0.1,0.2>\right\} ; \\
A_{6}=\left\{<x_{1}, 0.55,0.2,0.25>,<x_{2}, 0.57,0.2,0.15>\right\}
\end{gathered}
$$

Then we employ SVNMST clustering algorithm to group these operational plans $A_{j}(j=1,2, \ldots, 6)$.

Step 1. Calculate the distance $d_{i j}=d_{\lambda}\left(A_{i}, A_{j}\right)$ by Equation (1) (take $\left.\lambda=2\right)$. Then we can establish the singlevalued neutrosophic distance matrix $D=\left(d_{i j}\right)_{m \times m}$ as follows:

$$
D=\left[\begin{array}{cccccc}
0.0000 & 0.2327 & 0.1507 & 0.1637 & 0.1940 & 0.1051 \\
0.2327 & 0.0000 & 0.1127 & 0.1326 & 0.1131 & 0.1546 \\
0.1507 & 0.1127 & 0.0000 & 0.1056 & 0.0764 & 0.0802 \\
0.1637 & 0.1326 & 0.1056 & 0.0000 & 0.0940 & 0.0784 \\
0.1940 & 0.1131 & 0.0764 & 0.0940 & 0.0000 & 0.1210 \\
0.1051 & 0.1546 & 0.0802 & 0.0784 & 0.1210 & 0.0000
\end{array}\right] .
$$

Table 1. SVNMST Clustering Results of the Eight Different Cars.

\begin{tabular}{ll}
\hline$r$ & Corresponding to clustering result \\
\hline$r=d_{18}=0.41$ & $\left\{A_{1}, A_{2}, A_{3}, A_{4}, A_{5}, A_{6}, A_{7}, A_{8}\right\}$ \\
$r=d_{15}=0.4022$ & $\left\{A_{1}, A_{2}, A_{3}, A_{4}, A_{5}, A_{6}, A_{7}\right\},\left\{A_{8}\right\}$ \\
$r=d_{37}=0.3983$ & $\left\{A_{1}, A_{2}, A_{3}, A_{4}, A_{7}\right\},\left\{A_{5}, A_{6}\right\},\left\{A_{8}\right\}$ \\
$r=d_{24}=0.2605$ & $\left\{A_{1}, A_{2}, A_{3}, A_{4}\right\},\left\{A_{5}, A_{6}\right\},\left\{A_{7}\right\},\left\{A_{8}\right\}$ \\
$r=d_{13}=0.2551$ & $\left\{A_{1}, A_{2}, A_{3}\right\},\left\{A_{4}\right\},\left\{A_{5}, A_{6}\right\},\left\{A_{7}\right\},\left\{A_{8}\right\}$ \\
$r=d_{23}=0.2269$ & $\left\{A_{1}\right\},\left\{A_{2}, A_{3}\right\},\left\{A_{4}\right\},\left\{A_{5}, A_{6}\right\},\left\{A_{7}\right\},\left\{A_{8}\right\}$ \\
$r=d_{56}=0.1987$ & $\left\{A_{1}\right\},\left\{A_{2}\right\},\left\{A_{3}\right\},\left\{A_{4}\right\},\left\{A_{5}, A_{6}\right\},\left\{A_{7}\right\},\left\{A_{8}\right\}$ \\
$r=0$ & $\left\{A_{1}\right\},\left\{A_{2}\right\},\left\{A_{3}\right\},\left\{A_{4}\right\},\left\{A_{5}\right\},\left\{A_{6}\right\},\left\{A_{7}\right\},\left\{A_{8}\right\}$ \\
\hline
\end{tabular}


Step 2. Draw the single-valued neutrosophic graph $G(V, E)$ where every edge between $A_{i}$ and $A_{j}(i, j=1,2, \ldots, 6)$ is assigned the weight (single-valued neutrosophic distance) $d_{i j}$ coming from an element of the singlevalued neutrosophic distance matrix $D=\left(d_{i j}\right)_{m \times m}$, which represents the dissimilarity degree between the samples $A_{i}$ and $A_{j}$. The single-valued neutrosophic graph $G(V, E)$ is shown in Figure 6.

Step 3. Build the MST of the single-valued neutrosophic graph $G(V, E)$ by Kruskal's method [5] or Prim's method [6].

1. Sort the edges of $G$ in increasing order by weights:

$$
d_{35}<d_{46}<d_{36}<d_{45}<d_{16}<d_{34}<d_{23}<d_{25}<d_{56}<d_{24}<d_{13}<d_{26}<d_{14}<d_{15}<d_{12} .
$$

2. Keep an empty subgraph $S$ of $G$ and add the edge $e$ with the smallest weight to $S$, where the end points of $e$ are disconnected; thus, we choose the edge $e_{35}$ between $A_{3}$ and $A_{5}$.

3. Repeat process (2) until the subgraph $S$ spans six nodes. Thus, the MST of the single-valued neutrosophic graph $G(V, E)$ is obtained, as shown in Figure 7.

Step 4. Select a threshold $r$ and disconnect all the edges of the MST with weights greater than $r$ to obtain a certain number of subtrees (clusters), as listed in Table 2.

To compare the SVNMST clustering algorithm with the IFMST clustering algorithm in [15], assume that the indeterminacy membership function $I_{A}\left(x_{i}\right)$ is not considered independently in an SVNS $A_{i}$, then the samples' information given by the military committee will be the intuitionistic fuzzy data (adopted in [15]):

$$
A_{1}=\left\{\left\langle x_{1}, 0.7,0.15\right\rangle,\left\langle x_{2}, 0.6,0.2>\right\}, A_{2}=\left\{\left\langlex_{1}, 0.4,0.35>,\left\langle x_{2}, 0.8,0.1>\right\} ;\right.\right.\right.
$$

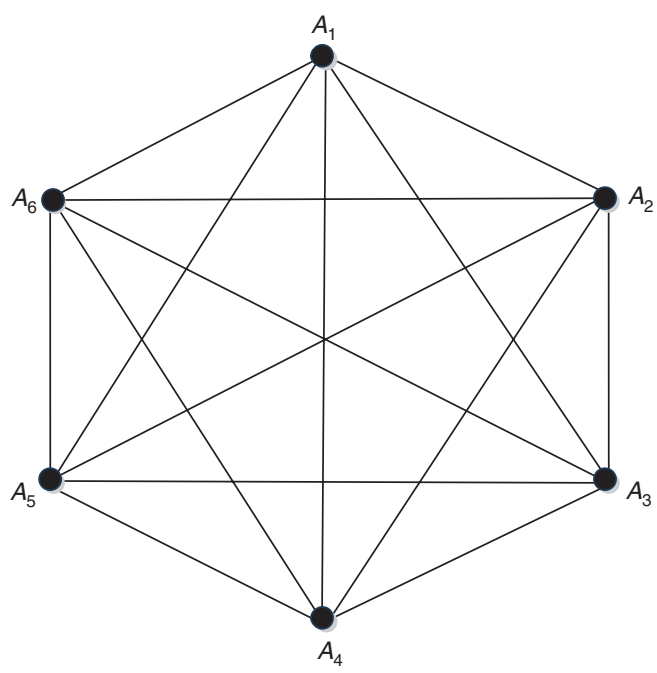

Figure 6. Single-Valued Neutrosophic Graph $G(V, E)$ with the Six Nodes.

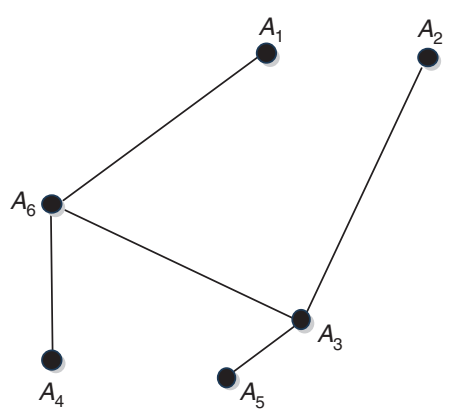

Figure 7. The MST of the Single-Valued Neutrosophic Graph $G(V, E)$ with the Six Nodes. 
Table 2. SVNMST Clustering Results of the Six Operational Plans.

\begin{tabular}{ll}
\hline$r$ & Corresponding to clustering result \\
\hline$r=d_{23}=0.1127$ & $\left\{A_{1}, A_{2}, A_{3}, A_{4}, A_{5}, A_{6}\right\}$ \\
$r=d_{16}=0.1051$ & $\left\{A_{2}\right\},\left\{A_{1}, A_{3}, A_{4}, A_{5}, A_{6}\right\}$ \\
$r=d_{36}=0.0802$ & $\left\{A_{1}\right\},\left\{A_{2}\right\},\left\{A_{3}, A_{4}, A_{5}, A_{6}\right\}$ \\
$r=d_{46}=0.0784$ & $\left\{A_{1}\right\},\left\{A_{2}\right\},\left\{A_{3}, A_{5}\right\},\left\{A_{4}, A_{6}\right\}$ \\
$r=d_{35}=0.0764$ & $\left\{A_{1}\right\},\left\{A_{2}\right\},\left\{A_{3}, A_{5}\right\},\left\{A_{4}\right\},\left\{A_{6}\right\}$ \\
$r=0$ & $\left\{A_{1}\right\},\left\{A_{2}\right\},\left\{A_{3}\right\},\left\{A_{4}\right\},\left\{A_{5}\right\},\left\{A_{6}\right\}$ \\
\hline
\end{tabular}

$$
\begin{aligned}
& A_{3}=\left\{<x_{1}, 0.55,0.25>,<x_{2}, 0.7,0.15>\right\}, A_{4}=\left\{<x_{1}, 0.44,0.35>,<x_{2}, 0.6,0.2>\right\} \\
& A_{5}=\left\{<x_{1}, 0.5,0.35>,<x_{2}, 0.75,0.2>\right\}, A_{6}=\left\{<x_{1}, 0.55,0.25>,<x_{2}, 0.57,0.15>\right\}
\end{aligned}
$$

Then, the operational plans $A_{j}(j=1,2, \ldots, 6)$ can be clustered by the following IFMST clustering algorithm [15].

Step 1. Calculate $d_{i j}=d\left(A_{i}, A_{j}\right)$ by the intuitionistic fuzzy distance measure:

$$
d(A, B)=\left\{\frac{1}{2} \sum_{i=1}^{n} w_{i}\left[\left|\mu_{A}\left(x_{i}\right)-\mu_{B}\left(x_{i}\right)\right|^{2}+\left|v_{A}\left(x_{i}\right)-v_{B}\left(x_{i}\right)\right|^{2}+\left|\pi_{A}\left(x_{i}\right)-\pi_{B}\left(x_{i}\right)\right|^{2}\right]\right\}^{1 / 2} .
$$

Thus, we get the intuitionistic fuzzy distance matrix:

$$
D=\left[\begin{array}{cccccc}
0.0000 & 0.2450 & 0.1225 & 0.1170 & 0.1725 & 0.1115 \\
0.2450 & 0.0000 & 0.1225 & 0.1280 & 0.1000 & 0.1940 \\
0.1225 & 0.1225 & 0.0000 & 0.1045 & 0.1000 & 0.0715 \\
0.1170 & 0.1280 & 0.1045 & 0.0000 & 0.1095 & 0.0935 \\
0.1725 & 0.1000 & 0.1000 & 0.1095 & 0.0000 & 0.1715 \\
0.1115 & 0.1940 & 0.0715 & 0.0935 & 0.1715 & 0.0000
\end{array}\right] \text {. }
$$

Step 2. Structuring the intuitionistic fuzzy graph $G=(V, E)$, see Step 2 in the SVNMST clustering algorithm and Figure 6.

Step 3. Get the MST of the intuitionistic fuzzy graph $G=(V, E)$ by Kruskal's method [5] or Prim's method [6]:

1. Sort the edges of $G$ in increasing order by weights:

$$
d_{36}<d_{46}<d_{35}=d_{25}<d_{34}<d_{45}<d_{16}<d_{14}<d_{13}=d_{23}<d_{24}<d_{56}<d_{15}<d_{26}<d_{52} \text {. }
$$

2. Keep an empty subgraph $S$ of $G$ and add the edge $e$ with the smallest weight to $S$, where the end points of $e$ are disconnected; thus, we choose the edge $e_{36}$ between $A_{3}$ and $A_{6}$.

3. Repeat process (2) until the subgraph $S$ spans six nodes. Thus, the MST of the intuitionistic fuzzy graph $G(V, E)$ is obtained, as shown in Figure 8.

Step 4. Select a threshold $r$ and disconnect all the edges of the MST with weights greater than $r$ to obtain a certain number of subtrees (clusters), as listed in Table 3.

It is well known that the fuzzy set is only composed of the membership degree. Therefore, we only consider the membership degrees of intuitionistic fuzzy data, and then the operational plan information given by the following fuzzy data [15]:

$$
\begin{gathered}
A_{1}=\left\{\left\langle x_{1}, 0.7>,<x_{2}, 0.6>\right\}, A_{2}=\left\{\left\langle x_{1}, 0.4>,<x_{2}, 0.8>\right\}, A_{3}=\left\{<x_{1}, 0.55>,<x_{2}, 0.7>\right\} ;\right.\right. \\
A_{4}=\left\{<x_{1}, 0.44>,<x_{2}, 0.6>\right\}, A_{5}=\left\{<x_{1}, 0.5>,<x_{2}, 0.75>\right\}, A_{6}=\left\{<x_{1}, 0.55>,<x_{2}, 0.57>\right\} .
\end{gathered}
$$




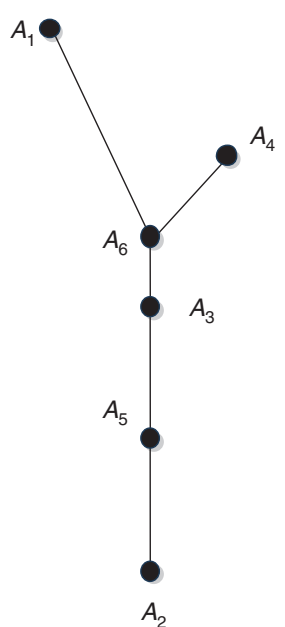

Figure 8. The MST of the Intuitionistic Fuzzy Graph $G=(V, E)$.

Then, the operational plans $A_{j}(j=1,2, \ldots, 6)$ can be clustered by the fuzzy MST clustering algorithm: Step 1. Calculate $d_{i j}=d\left(A_{i}, A_{j}\right)$ by the fuzzy distance measure:

$$
d(A, B)=\left\{\sum_{i=1}^{2} w_{i}\left[\left|\mu_{A}\left(x_{i}\right)-\mu_{B}\left(x_{i}\right)\right|^{2}\right]\right\}^{1 / 2} .
$$

Thus, we obtain the fuzzy distance matrix:

$$
D=\left[\begin{array}{cccccc}
0.0000 & 0.2500 & 0.1250 & 0.1744 & 0.1743 & 0.1031 \\
0.2500 & 0.0000 & 0.1250 & 0.1507 & 0.0766 & 0.1980 \\
0.1250 & 0.1250 & 0.0000 & 0.1046 & 0.0500 & 0.0964 \\
0.1744 & 0.1507 & 0.1046 & 0.0000 & 0.1183 & 0.0771 \\
0.1743 & 0.0766 & 0.0500 & 0.1183 & 0.0000 & 0.1376 \\
0.1031 & 0.1980 & 0.0964 & 0.0771 & 0.1376 & 0.0000
\end{array}\right] .
$$

Step 2. Draw the fuzzy graph $G=(V, E)$. See also Step 2 in the SVNMST clustering algorithm and Figure 6 . Step 3. Build the MST of the fuzzy graph $G=(V, E)$ by Kruskal's method [5] or Prim's method [6]:

1. Sort the edges of $G$ in increasing order by weights: $d_{35}<d_{25}<d_{46}<d_{36}<d_{16}<d_{34}<d_{45}<d_{13}=d_{23}<d_{56}<d_{24}<d_{15}<d_{14}<d_{26}<d_{12}$.

2. Keep an empty subgraph $S$ of $G$ and add the edge $e$ with the smallest weight to $S$, where the end points of $e$ are disconnected; thus, we choose the edge $e_{35}$ between $A_{3}$ and $A_{5}$.

3. Repeat process (2) until the subgraph $S$ spans six nodes. Thus, the MST of the fuzzy graph $G(V, E)$ is obtained, as shown in Figure 9.

Table 3. IFMST Clustering Results of the Six Operational Plans.

\begin{tabular}{ll}
\hline$r$ & Corresponding to clustering result \\
\hline$r=d_{16}=0.1115$ & $\left\{A_{1}, A_{2}, A_{3}, A_{4}, A_{5}, A_{6}\right\}$ \\
$r=d_{25}=d_{35}=0.1$ & $\left\{A_{1}\right\},\left\{A_{2}, A_{3}, A_{4}, A_{5}, A_{6}\right\}$ \\
$r=d_{46}=0.088$ & $\left\{A_{1}\right\},\left\{A_{2}\right\},\left\{A_{5}\right\},\left\{A_{3}, A_{4}, A_{6}\right\}$ \\
$r=d_{36}=0.0715$ & $\left\{A_{1}\right\},\left\{A_{2}\right\},\left\{A_{4}\right\},\left\{A_{5}\right\},\left\{A_{3}, A_{6}\right\}$ \\
$r=0$ & $\left\{A_{1}\right\},\left\{A_{2}\right\},\left\{A_{3}\right\},\left\{A_{4}\right\},\left\{A_{5}\right\},\left\{A_{6}\right\}$ \\
\hline
\end{tabular}




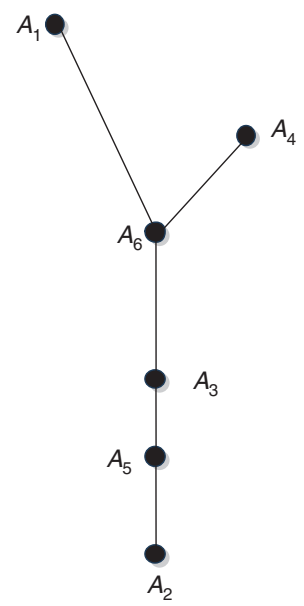

Figure 9. The MST of the Fuzzy Graph $G=(V, E)$.

Step 4. Select a threshold $r$ and disconnect all the edges of the MST with weights greater than $r$ to obtain a certain number of subtrees (clusters), as listed in Table 4.

For convenient comparisons, we put the clustering results of three kinds of clustering algorithms into Table 5.

From Table 5, we can see that the clustering results of the three clustering algorithms are quite different. The main reason can be given by the following comparative analysis.

As is known to all, the single-valued neutrosophic information is a generalization of intuitionistic fuzzy information, and intuitionistic fuzzy information is a further generalization of fuzzy information. On the one hand, an SVNS is an instance of a neutrosophic set, which gives us an additional possibility to represent uncertainty, imprecise, incomplete, and inconsistent information, which exist in real world. It can describe and handle indeterminate information and inconsistent information. However, the connector in the fuzzy set is defined with respect to $T$, i.e., membership only, hence the information of indeterminacy and nonmembership is lost. The connectors in the intuitionistic fuzzy set are defined with respect to $T$ and $F$, i.e., membership and nonmembership only; hence, the indeterminacy is what is left from 1 and then the intuitionistic fuzzy set can only handle incomplete information but not the indeterminate information and inconsistent information, whereas in the SVNS, its truth membership, indeterminacy membership, and falsity membership are represented independently, and then they can be defined with respect to any of them (no restriction). Therefore, the notion of SVNSs is more general. On the other hand, the clustering analysis under single-valued neutrosophic environment is suitable for capturing imprecise, uncertain, and inconsistent information in clustering the data. Thus, the SVNMST clustering algorithm clusters the single-valued neutrosophic information, whereas the IFMST clustering algorithm clusters the intuitionistic fuzzy information and the fuzzy MST clustering algorithm clusters the fuzzy information. Obviously, the SVNMST clustering algorithm is the extension of both the IFMST clustering algorithm and the fuzzy MST clustering algorithm. Therefore, compared with the IFMST clustering algorithm and the fuzzy MST clustering algorithm, the SVNMST clustering

Table 4. Fuzzy MST Clustering Results.

\begin{tabular}{ll}
\hline$r$ & Corresponding to clustering result \\
\hline$r=d_{16}=0.1031$ & $\left\{A_{1}, A_{2}, A_{3}, A_{4}, A_{5}, A_{6}\right\}$ \\
$r=d_{36}=0.0964$ & $\left\{A_{1}\right\},\left\{A_{2}, A_{3}, A_{4}, A_{5}, A_{6}\right\}$ \\
$r=d_{46}=0.0774$ & $\left\{A_{1}\right\},\left\{A_{2}, A_{3}, A_{5}\right\},\left\{A_{4}, A_{6}\right\}$ \\
$r=d_{25}=0.0766$ & $\left\{A_{1}\right\},\left\{A_{2}, A_{3}, A_{5}\right\},\left\{A_{4}\right\},\left\{A_{6}\right\}$ \\
$r=d_{35}=0.0500$ & $\left\{A_{1}\right\},\left\{A_{2}\right\},\left\{A_{3}, A_{5}\right\},\left\{A_{4}\right\},\left\{A_{6}\right\}$ \\
$r=0$ & $\left\{A_{1}\right\},\left\{A_{2}\right\},\left\{A_{3}\right\},\left\{A_{4}\right\},\left\{A_{5}\right\},\left\{A_{6}\right\}$ \\
\hline
\end{tabular}


Table 5. Clustering Results of Three Kinds of Clustering Algorithms.

\begin{tabular}{clll}
\hline Class & SVNMST clustering algorithm & IFMST clustering algorithm & Fuzzy MST clustering algorithm \\
\hline 1 & $\left\{A_{1}, A_{2}, A_{3}, A_{4}, A_{5}, A_{6}\right\}$ & $\left\{A_{1}, A_{2}, A_{3}, A_{4}, A_{5}, A_{6}\right\}$ & $\left\{A_{1}, A_{2}, A_{3}, A_{4}, A_{5}, A_{6}\right\}$ \\
2 & $\left\{A_{2}\right\},\left\{A_{1}, A_{3}, A_{4}, A_{5}, A_{6}\right\}$ & $\left\{A_{1}\right\},\left\{A_{2}, A_{3}, A_{4}, A_{5}, A_{6}\right\}$ & $\left\{A_{1}\right\},\left\{A_{2}, A_{3}, A_{4}, A_{5}, A_{6}\right\}$ \\
3 & $\left\{A_{1}\right\},\left\{A_{2}\right\},\left\{A_{3}, A_{4}, A_{5}, A_{6}\right\}$ & & $\left\{A_{1}\right\},\left\{A_{2}, A_{3}, A_{5}\right\},\left\{A_{4}, A_{6}\right\}$ \\
4 & $\left\{A_{1}\right\},\left\{A_{2}\right\},\left\{A_{3}, A_{5}\right\},\left\{A_{4}, A_{6}\right\}$ & $\left\{A_{1}\right\},\left\{A_{2}\right\},\left\{A_{5}\right\},\left\{A_{3}, A_{4}, A_{6}\right\}$ & $\left\{A_{1}\right\},\left\{A_{2}, A_{3}, A_{5}\right\},\left\{A_{4}\right\},\left\{A_{6}\right\}$ \\
5 & $\left\{A_{1}\right\},\left\{A_{2}\right\},\left\{A_{3}, A_{5}\right\},\left\{A_{4}\right\},\left\{A_{6}\right\}$ & $\left\{A_{1}\right\},\left\{A_{2}\right\},\left\{A_{4}\right\},\left\{A_{5}\right\},\left\{A_{3}, A_{6}\right\}$ & $\left\{A_{1}\right\},\left\{A_{2}\right\},\left\{A_{3}, A_{5}\right\},\left\{A_{4}\right\},\left\{A_{6}\right\}$ \\
6 & $\left\{A_{1}\right\},\left\{A_{2}\right\},\left\{A_{3}\right\},\left\{A_{4}\right\},\left\{A_{5}\right\},\left\{A_{6}\right\}$ & $\left\{A_{1}\right\},\left\{A_{2}\right\},\left\{A_{3}\right\},\left\{A_{4}\right\},\left\{A_{5}\right\},\left\{A_{6}\right\}$ & $\left\{A_{1}\right\},\left\{A_{2}\right\},\left\{A_{3}\right\},\left\{A_{4}\right\},\left\{A_{5}\right\},\left\{A_{6}\right\}$ \\
\hline
\end{tabular}

algorithm is more general. Furthermore, when we encounter some situations, which are represented by indeterminate information and inconsistent information, the SVNMST clustering algorithm demonstrates its great superiority in clustering those single-valued neutrosophic data.

\section{Conclusion}

This article defined a generalized single-valued neutrosophic weighted distance and proposed the SVNMST clustering algorithm as a generalization of the IFMST clustering algorithm. Through the computational tests on the SVNMST clustering algorithm, the IFMST clustering algorithm, and the fuzzy MST clustering algorithm, the clustering results have shown that the SVNMST clustering algorithm is more general and more reasonable than the IFMST clustering algorithm and the fuzzy MST clustering algorithm. Furthermore, in situations that are represented by indeterminate information and inconsistent information, the SVNMST clustering algorithm demonstrates its great superiority in clustering those single-valued neutrosophic data because the SVNSs are a powerful tool to deal with uncertainty, imprecise, incomplete, and inconsistent information. In the future, the developed algorithm can be applied to many areas such as information retrieval, investment decision making, and data mining.

Received September 25, 2013; previously published online January 8, 2014.

\section{Bibliography}

[1] D. S. Chen, K. X. Li and L. B. Zhao, Fuzzy graph maximal tree clustering method and its application, Oper. Res. Manage. Sci. 16 (2007), 69-73.

[2] Y. H. Dong, Y. T. Zhuang, K. Chen and X. Y. Tu, A hierarchical clustering algorithm based on fuzzy graph connectedness, Fuzzy Sets Syst. 157 (2006), 1760-1774.

[3] J. Harary, Graph theory, Addison-Wesley, Reading, 1969.

[4] A. K. Jain and R. C. Dubes, Algorithms for clustering data, Prentice-Hall, Englewood Cliffs, NJ, 1988.

[5] J. B. Kruskal, On the shortest spanning subtree of a graph and the traveling salesman problem, Proc. Am. Math. Soc. 7 (1956), 48-50.

[6] R. C. Prim, Shortest connection networks and some generalizations, Bell Syst. Technol. J. 36 (1957), 1389-1401.

[7] E. H. Ruspini, A new approach to clustering. Inform. Control 15 (1969), 22-32.

[8] F. Smarandache, A unifying field in logics. Neutrosophy: neutrosophic probability, set and logic, American Research Press, Rehoboth, 1998.

[9] H. Wang, F. Smarandache, Y. Q. Zhang and R. Sunderraman, Single valued neutrosophic sets, Multispace Multistruct. 4 (2010), 410-413.

[10] Y. Xu, V. Olman and D. Xu, Clustering gene expression data using a graph-theoretic approach: an application of minimum spanning tree, Bioinformatics 18 (2002), 536-545.

[11] J. Ye, Multicriteria decision-making method using the correlation coefficient under single-valued neutrosophic environment, Int. J. Gen. Syst. 42 (2013), 386-394. 
[12] J. Ye, Single valued neutrosophic cross-entropy for multicriteria decision making problems, Appl. Math. Model. (2013) doi: 10.1016/j.apm.2013.07.020.

[13] C. T. Zahn, Graph-theoretical methods for detecting and describing gestalt clusters, IEEE Trans. Comput. C 20 (1971), 68-86.

[14] X. Zhang and Z. Xu, An MST cluster analysis method under hesitant fuzzy environment, Control Cybern. 41 (2012), $645-666$.

[15] H. Zhao, Z. Xu, S. Liu and Z. Wang, Intuitionistic fuzzy MST clustering algorithms, Comput. Indust. Eng. 62 (2012), $1130-1140$. 